\title{
Postural control during a transition task in haemophilic children, adolescents and young adults with haemophilic ankle arthropathy
}

\author{
K. Deschamps $s^{1,2,3,4}$ (D) | F. Staes ${ }^{1} \mid$ M. Eerdekens ${ }^{4}$ | K. Peerlinck ${ }^{5}$ | C. Hermans ${ }^{6}$ | \\ J. Vandesande ${ }^{5}$ | S. Lobet ${ }^{6,7,8}$ (i)
}

${ }^{1}$ Department of Rehabilitation Sciences, Musculoskeletal Rehabilitation Research Group, KULeuven, Leuven (Heverlee), Belgium

${ }^{2}$ Division of Podiatry, Institut

D'Enseignement, Supérieur Parnasse DeuxAlice, Sint-Lambrechts-Woluwe, Belgium

${ }^{3}$ Department of Podiatry, Artevelde University College, Ghent, Belgium

${ }^{4}$ Department of Physical Medicine, University Hospitals Leuven, Pellenberg, Belgium

${ }^{5}$ Department of Cardiovascular Sciences, Centre for Molecular and Vascular Biology, KULeuven, Leuven, Belgium

${ }^{6}$ Service d'hématologie, Cliniques Universitaires Saint-Luc, Bruxelles, Belgium

${ }^{7}$ Secteur des Sciences de la Santé, Institut de Recherche Expérimentale et

Clinique, Neuromusculoskeletal Lab (NMSK), Université catholique de Louvain, Brussels, Belgium

${ }^{8}$ Service de médecine physique et réadaptation, Cliniques universitaires SaintLuc, Brussels, Belgium

Correspondence

Kevin Deschamps, Department of

Rehabilitation Sciences- Musculoskeletal

Rehabilitation Research Group, KULeuven, Heverlee, Belgium.

Email: kevin.deschamps@faber.kuleuven.be

Funding information

Bayer HealthCare, Grant/Award Number: Early Career Investigator Award 2012; Pfizer, Grant/Award Number: ASPIRE 2013; World Federation of Haemophilia Clinical Research Grant Program; Bayer Hemophilia Awards Program
Background: The aim of this study was to determine whether young haemophilic boys with and without MRI-based signs of ankle arthropathy demonstrate reduced balance ability during a transition task with eyes open and eyes closed.

Methods: Thirty-four haemophilic bodies and 28 typically developing boys aged 6-20 years participated to this study. Structural integrity of the tarsal foot joints of all haemophilic boys was assessed with MRI. All participants performed a standard transition task from double-leg stance to single-leg stance with eyes open and eyes closed. Comparison of balance features derived from the centre of pressure displacement captured by a single force platform was performed between the different haemophilia subgroups and sex-age-height matched peers.

Findings: The haemophilic boys without signs of arthropathy presented only a higher intermediate phase velocity during the eyes closed condition $(P=.05)$. The haemophilic boys with signs of arthropathy had significantly higher displacement after the time to new stability point, and 95\% Ellipse Sway Area and Balance Area compared to their matched peers during eyes open test $(P<.05)$. Similar findings were observed during the eyes closed test for the displacement after the time to new stability point and 95\% Ellipse Sway Area $(P<.05)$. No significant differences were observed between affected and non-affected side of the unilateral affected patients.

Interpretation: We suggest that the pathophysiological cascade associated with chronic bleeding episodes should not be considered as a "simple" musculoskeletal injury, hence more as a complex neurophysiological dysfunction which may originate both from unilateral and bilateral deterioration of the musculoskeletal system.

\section{KEYWORDS}

ankle arthropathy, balance, Haemophiliac, postural control 


\section{1 | INTRODUCTION}

Haemophiliac is a rare $X$ chromosome-linked coagulation disorder resulting from a congenital deficiency or the absence of circulating factor VIII (Haemophilia A) or factor IX (haemophilia B). Consequently, patients with haemophiliac are unable to generate adequate thrombin resulting in abnormal bleeding. ${ }^{1}$ Approximately $80 \%-90 \%$ of bleeding episodes occur in the musculoskeletal system, especially in the elbows, knees and ankles, and in the muscles. Repeated haemarthroses induce joint cartilage damage and irreversible degenerative joint disease called haemophilic arthropathy. ${ }^{2}$ Regular intravenous administration of coagulation factor concentrates starting after the first joint bleed and/or before the age of 2 ("primary" prophylaxis) is now the evidence-based, first-choice treatment in children with severe haemophiliac. ${ }^{3}$ This primary prophylaxis has radically decreased the incidence of haemophilic arthropathy. Despite this positive evolution, the ankle region constituted of the tibiotalar joint (TTJ) and subtalar joint (STJ) seems to be an exception to the rule, as children with haemophiliac treated with primary prophylaxis still experience TTJ and/or STJ arthropathy. As a consequence, these two linked joints are nowadays considered to be the clinically most indicative joint in children with haemophiliac. ${ }^{4}$

Because haemophilic arthropathy is characterized by a very specific pathophysiological process or cascade ${ }^{2}$ and because there is a lack of remedy, it is imperative to understand the impairments associated with early forms of arthropathy. Scientific literature describes the relationship between certain articular pathologies and postural instability. ${ }^{5,6}$ It is reasonable to assume that doing the same in the perspective of the TTJ and STJ haemophilic arthropathy pathophysiological cascade in children and adolescents with haemophiliac may be of clinical, sociologic and economic interest. To our knowledge, only five studies investigated postural control performance in patients with haemophiliac; two in adults ${ }^{7,8}$ and two in children with haemophiliac. ${ }^{9,10}$ The fifth study by Hilberg et al. ${ }^{11}$ included a haemophilic population with an age range between 16 and 44 years. Comparing the results of these studies is difficult, as there exists considerable methodological variation. However, in general, poorer postural control and greater postural disharmonies were observed in the haemophilic patient groups when compared to non-haemophilic peers, leading to the general statement that balance measures should be integrated into routine clinical practice. Critical appraisal of the literature reveals that a comprehensive perspective on the relationship between haemophilic ankle arthropathy and postural balance is lacking.

First, previously mentioned studies omitted a robust quantification of the TTJ and STJ integrity. Joint structural integrity in these studies was assessed with disease-specific clinical scores (eg HJHS, World Federation of Haemophilia clinical score). However, these scores show good sensitivity with respect to the detection of early stages of ankle arthropathy but not for other major foot joints such as the subtalar joint. ${ }^{12}$ Thus, the relationship between HA and postural control remains poorly studied. Signs or early manifestation of $\mathrm{HA}$ is associated with altered postural control. If present, any balance deficits may deteriorate further within the aforementioned cascade. Such evidence may then inform the development of identification strategies for individuals with HA on one hand, but may also help in the decision-making process with respect to the engagement in sports, physiotherapy and exercise regimens on the other hand.

Second, examination of balance control during a transition task from double-leg stance (DLS) to single-leg stance (SLS) has not been performed in children and adolescents with haemophiliac. During this task, one has to overcome the internal perturbation created by the shift to a smaller base of support. The challenging nature of such transition task is believed to be much higher compared to conventional static DLS and SLS, and it has the potential to be more sensitive to uncover dynamic balance deficits.

Therefore, the aim of this study was to determine whether children and adolescents with haemophiliac with and without MRI-based signs of ankle arthropathy demonstrate reduced balance ability during a transition task with eyes open (EO) and eyes closed (EC). We hypothesized reduced balance ability in patients with arthropathy and further hypothesized reduced balance ability between the affected and the non-affected sides in patients with unilateral involvement.

\section{2 | MATERIALS AND METHODS}

\section{1 | Participants}

This multi-centre study was approved by the Ethics Committee of the University Hospitals Leuven as well as the Ethics Committee of the Cliniques Universitaires Saint-Luc (S55957- ML9883B403201317010). Written informed consent was obtained for each study participant before undertaking any assessment. Study data were collected in a group of middle-aged children, adolescents and young adults (age between 6 and 20 years) with severe or moderate haemophiliac (Factor concentrate $<0.01$ and $0.01-0.05 \mathrm{IU} / \mathrm{mL}$, respectively) and regularly followed up at either of both Hospitals (Table 1). They were compared with a group of sex-age-height matched typically developing boys (TDB). Overall, 34 haemophilic boys ( 30 with haemophilia A and 4 with haemophilia B; 29 with severe and 5 with moderate haemophiliac) were included in the study and compared with 28 TDB.

The TDB had to meet the following criteria: (i) within 0.4th99.6th percentile for height and weight in relation to their chronological age, (ii) no gross gait abnormalities during visual inspection, (iii) free of major musculoskeletal trauma and (iv) free of surgery at the lower limbs.

\section{2 | Procedures}

\subsection{1 | Magnetic resonance imaging (MRI) assessment}

To obtain a state of the art evaluation of the structural integrity of the tarsal foot joints of all haemophilic patients, both TTJ and STJ were explored in a single MRI investigation using sagittal T1weighted spin-echo (SE) sequence, sagittal T2-weighted gradient 

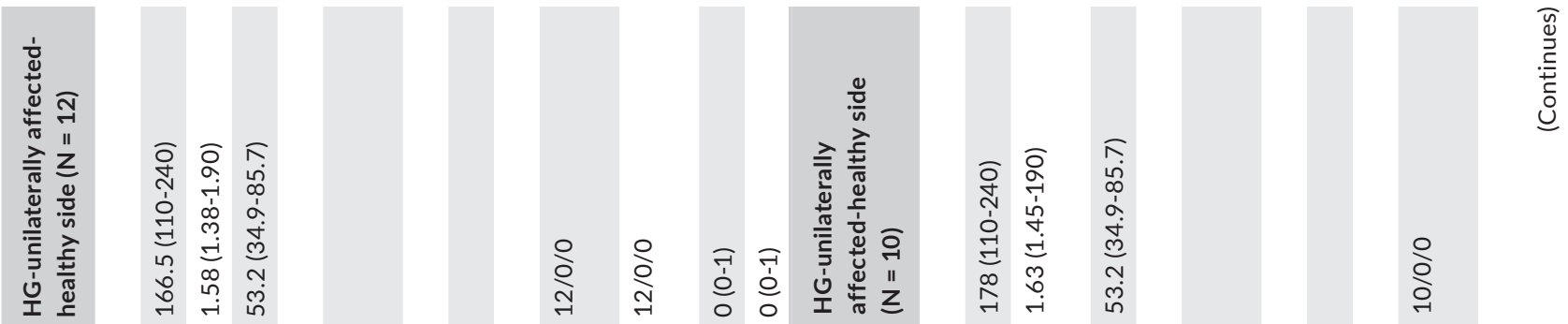

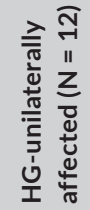

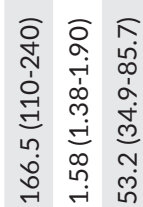

ำ $\stackrel{7}{0}$

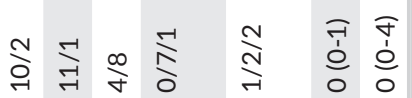

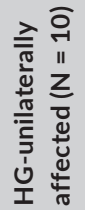

$\frac{0}{\frac{0}{2}}$

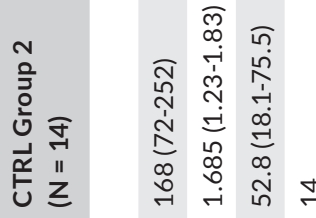

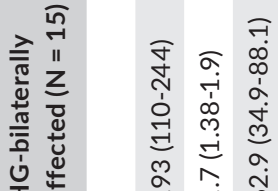

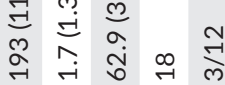

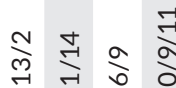

$\frac{0}{2}$

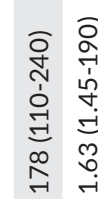

응

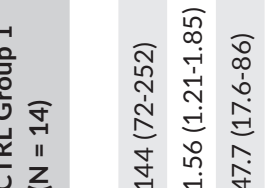

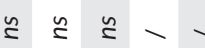

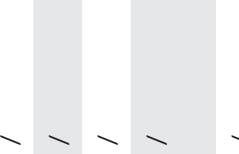

I

竎㭝余

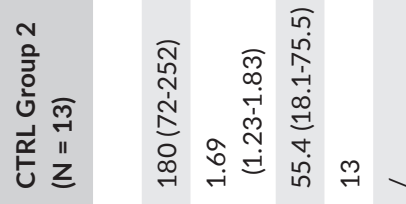

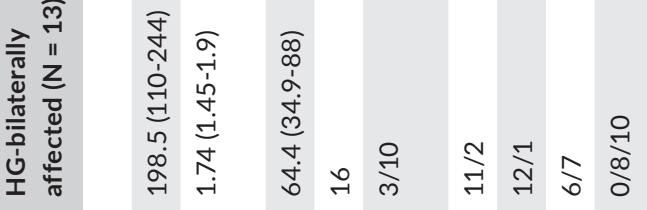

$\frac{2}{\frac{2}{2 \pi}}$

$\stackrel{\bar{\sigma}}{\bar{\sigma}}$

a

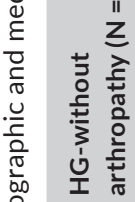

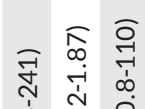

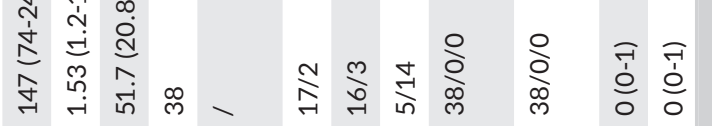

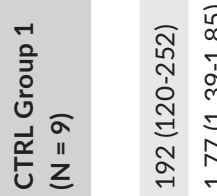

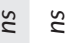

$\cong-$

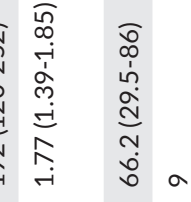




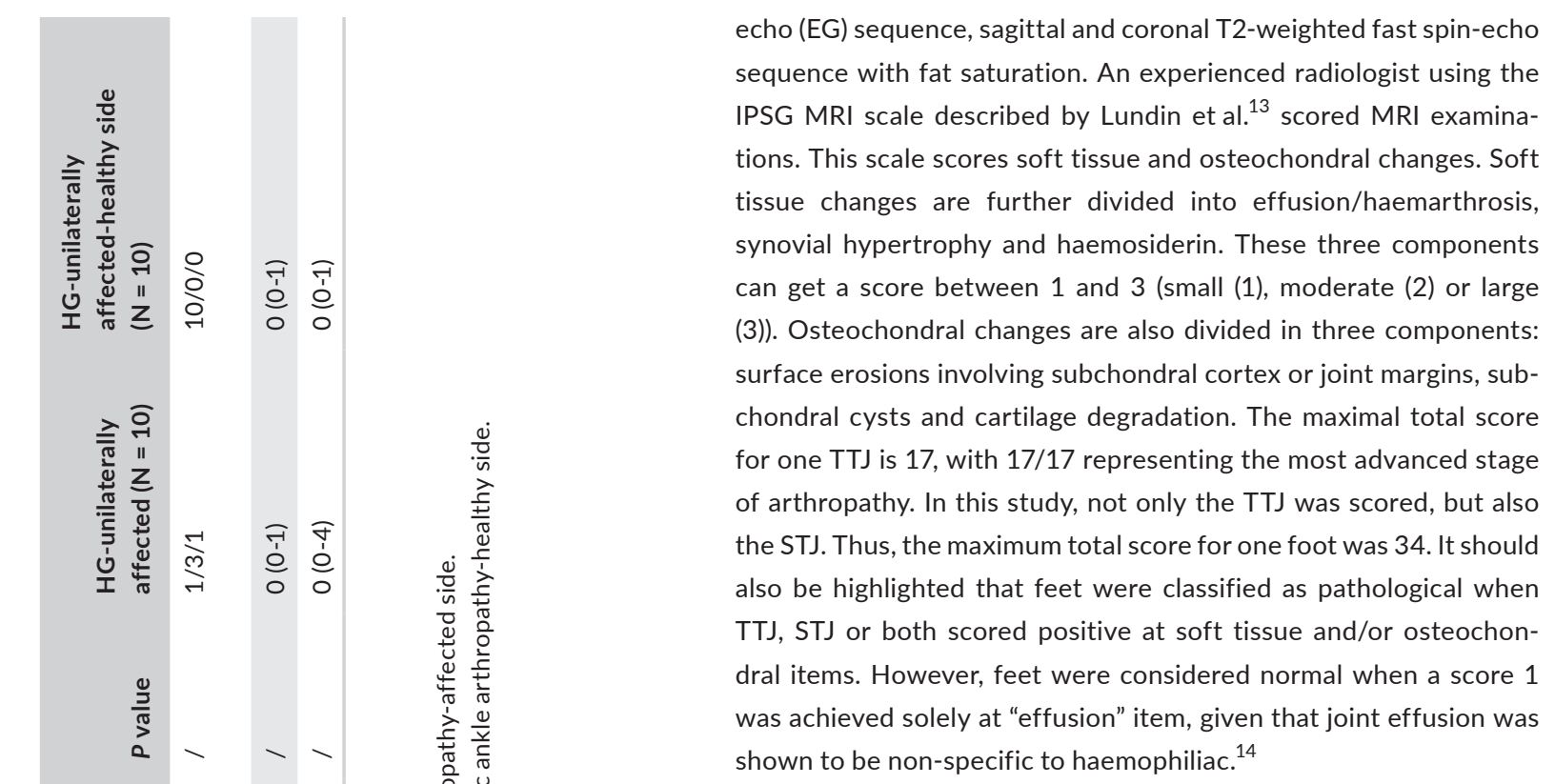

\subsubsection{Haemophilia joint health score (HJHS)}

Functional assessment was performed using the Haemophilia Joint Health Score version 2.1 (HJHS 2.1). The HJHS is a disease-specific 11-item score (swelling, duration, muscle atrophy, axial alignment, crepitus on motion, flexion loss, extension loss, instability, joint pain, strength and gait) that evaluates joint impairment of the ankle, knee and elbows in haemophilic patients. In this study, an experienced physiotherapist in the field of haemophiliac (SL) performed HJHS scoring of both knees and TTJ of each haemophilic boy.

\subsection{3 | Dynamic posturographic measurements}

Postural balance was assessed according to the methodology described by Dingenen et al. ${ }^{15}$ In brief, study participants were asked to stand on a force plate (Advanced Mechanical Technology, Watertown, US) with the feet separated by the width of the hips and the arms hanging loosely at the side. Each study participant performed a transition task from DLS to SLS. This was performed for both legs in a random sequence. Standardization of foot position between trials was assured by drawing the foot circumference on a paper lying on the aforementioned force plate.

The postural balance test started with a 15 seconds of DLS, followed by a movement towards one leg to try to stand on that leg for another 15 seconds (SLS). Participants were instructed to lift one leg, on the command of the examiner, towards approximately sixty degrees of hip flexion. Postural control performance during the transition task was tested at a preferred speed in two conditions: (i) with EO and (ii) with EC. The participants had to perform this task four times. During the test, participants were instructed to stare at a white wall. Before the actual measurements, all participants were allowed to familiarize with the transition task during two tryouts. The participants were allowed to rest between the trials to avoid fatigue. Analogue data were captured at $200 \mathrm{~Hz}$. 

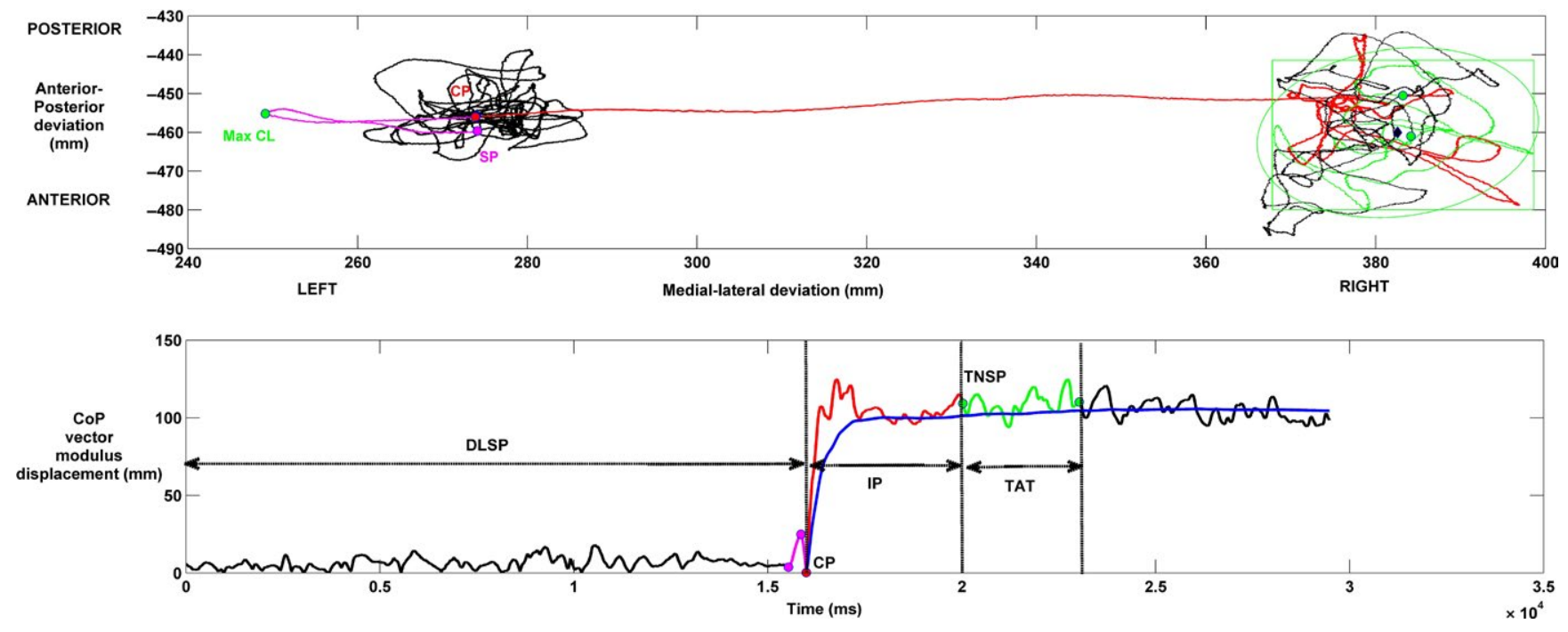

FIG URE 1 Top: Typical example of the two-dimensional centre of pressure displacement calculation during the transition from doubleleg stance to single-leg stance described by Dingenen et al. ${ }^{13}$ The black cloud at the left represents the COP trajectory during the doubleleg stance phase. The larger right cloud represents the COP trajectory during the single-leg stance phase. The COP excursion to the right represents the push-off movement to the contralateral side. SP: starting point of the contralateral push-off movement; Max CL: maximum contralateral COP excursion; CP: crossing-point; TNSP: time to new stability point. Green box and ellipse represent, respectively, the Balance Area (BA) and the 95\% Ellipse Sway Area (95\% ESA) specifically added in the current study. Bottom: An illustration of the modulus of the CoP vector (black) and the estimator (blue). DLSP: double-leg stance phase, CP: crossing-point, IP: intermediate phase, TNSP: Time to New Stability Point, TAT: CoP displacement during first $3 \mathrm{~s}$ after TNSP

\section{3 | Data extraction and analysis}

Data analysis associated with the postural control tasks was identical to that described by Dingenen et al. ${ }^{15} \mathrm{~A}$ brief description is provided below. First, the different task phases associated with the postural balance test were determined (Figure 1). During the task, a person moves first towards the opposite direction with a certain maximum deviation (contralateral push-off movement). ${ }^{15}$ Next, a movement is seen towards the single standing leg to finish the task. The time point, at which the medial-lateral $(\mathrm{M} / \mathrm{L})$ centre of pressure (CoP) crossed the average M/L CoP after the contralateral push-off movement, was defined as the crossing-point (CP). ${ }^{15}$ Starting from $\mathrm{CP}$, a sequential estimation method was used by calculating the cumulative average of the modulus of the CoP vector as basis for determining the time to the new stability point (TNSP). The 3-second time frame following the TNSP was subsequently determined (called TAT, Time After Time to new stability point). ${ }^{15}$ The total displacement of the CoP during this TAT was then calculated (TAT distance).

In addition, the 95\% Ellipse Sway Area (95\% ESA) and Balance Area (BA) were calculated during TAT. The former was defined as the $95 \%$ confidence ellipse of the CoP during TAT, whereas the latter embodies the total surface covered by the CoP during TAT. ${ }^{15}$

\section{4 | Statistical analysis}

For purposes of inferential comparison, the haemophilic cohort was subdivided based on MRI findings. Feet were classified as pathological when TTJ, STJ or both scored positive at soft tissue and/or osteochondral items. However, feet were considered normal when a score
1 was achieved solely for the "effusion" item, given that joint effusion was shown to be non-specific to haemophiliac. ${ }^{14}$ Consequently, subgroups were created in the haemophilic cohort. A group which was bilaterally affected (HG-bilaterally affected), a group which had no involvement (HG-without arthropathy) and a third group of patients which were unilaterally affected. The latter group was further subdivided into an "affected" (HG-unilaterally affected side) and a "non-affected group" (HG-unilaterally affected-healthy side).

A control group matching for sex-age (within 1 year)-height (5\%) was selected for the HG-without arthropathy (CTRL group 1) as well as for the HG-bilaterally affected (CTRL group 2). An independent Student's $t$ test was used to compare the dependent variables of the HG-without arthropathy and CTRL group 1, HG-bilaterally affected and CTRL group 2. Affected and healthy sides of the same subject were compared with a paired $t$ test. The alpha level was set to 0.05 . Data were analysed with Matlab 2012a (The Mathworks Inc., Natick, USA).

\section{3 | RESULTS}

Nineteen patients presented MRI anomalies. Unilateral damage was present in 12 patients and seven patients showed bilateral damage. In twenty-six affected ankles, isolated TTJ anomalies were observed in 15 cases, five had both TTJ and STJ anomalies and six had isolated STJ anomalies (Table 1).

The included number of participants varied across the EO and EC conditions. A number of dropouts were encountered for the EC balance test as it was found to be to challenging for the youngest 
participants (Table 1). Due to high variability in patient characteristics (especially body weight), it was not possible to have a unique matched control subject per patient, which led to a situation that a given control subject was used more than once for inferential purposes (Table 1).

The HG-without arthropathy had a higher IP velocity during the EC condition compared to the matched peers $(P=.05)$ (Table 2$)$.

The HG-bilaterally affected presented a significantly higher TAT_ distance (0.17 vs $0.13 \mathrm{~m}, P=.05$ ), 95\% ESA (688.3 vs $516.7 \mathrm{~mm}^{2}$, $P=.05$ ) and BA (730.7 vs $529.1 \mathrm{~mm}^{2}, P=.03$ ) compared to their matched peers during the EO tests. Similar findings were observed during the EC test for the TAT_distance and 95\% ESA $(P<.05)$ (Table 2).

Finally, no significant differences were observed between the HG-unilaterally affected and HG-unilaterally affected-healthy side.

\section{4 | DISCUSSION}

To our knowledge, this is the first study investigating the balance ability of in children and adolescents with haemophiliac with and without MRI-based signs of haemophilic ankle arthropathy.

A considerable number of in children and adolescents with haemophiliac showed MRI anomalies of the TTJ and/or STJ in the current study. These findings are in agreement with the growing body of evidence that the ankle joint is the primary joint affected by haemophilic arthropathy in children and adolescents with haemophiliac. ${ }^{4}$

Recurrent bleedings together with progressive joint damage, formation of synovial hypertrophy and osteochondral damage are believed to progressively alter joint mechanoreceptor information and therefore postural control in patients with haemophiliac. ${ }^{5,6}$ The integrity of the postural control arc can be further affected by bleeding episodes within muscles, which in his turn may deteriorate muscle properties and integrity of the muscle spindles. As such, the postural control system may be caught in a vicious circle, which is assumed to contribute to an insidious pattern of microtrauma and (re)injury. ${ }^{11}$ The current study seems to provide evidence for this cascade. In fact, the HG-without arthropathy cohort was found to perform, with exception to the IP velocity during eyes closed, similarly as their matched peers.

Contrarily, patients with structural or soft tissue lesions proven on MRI were found to perform worse than their matched peers. Of particular interest, here is the observation that the so-called healthy side (the side with no evidence of structural of soft tissue lesions on MRI) of unilaterally affected patients seems to perform similar to the affected side. To some extent, this finding may be surprising, as one would expect the healthy side to perform better than the affected side. However, the findings of the current study agree with the study of Dingenen et al. ${ }^{15}$ which assessed through an identical transition task of the functional status of $A C L$ injured patients. In that study, the injured legs were found to have poorer postural control than their healthy peers; however, no significant differences were found between the injured and non-injured leg of the anterior cruciate ligament injured group. The authors hypothesized that the mechanism underlying these observations may be related to a reorganization of the central nervous system. They postulated that the capacity of the central nervous system to reintegrate and dynamically select the most reliable sensory information is essential in the development of effective stabilization strategies after injury. To avoid asymmetric neural control, the central nervous system would alter the functional behaviour of the non-injured leg. ${ }^{16}$

Extrapolation of this theory to the current study would lead to the suggestion that the pathophysiological cascade associated with chronic bleeding episodes should not be considered as a "simple" musculoskeletal injury, hence more as a complex neurophysiological dysfunction which may originate both from unilateral and bilateral deterioration of the musculoskeletal system. However, before this assumption can be generally accepted, more research is needed.

Proprioception plays a crucial role in human movement control, which is fundamental for daily activities, exercise and sports. Soon after joint injury, muscle weakness is often evident even though there may have been no direct injury to the muscle. This has been called arthrogenous muscle weakness. If this weakness affects neuromuscular protective reflexes, the patient may be prone to reinjury. Clinicians are therefore interested in detecting early signs of balance impairment and muscle weakness. In patients with haemophiliac, proprioceptive exercises are, in general, initiated as soon as possible following the acute hemarthrosis for instance. Efficacy of functional, closed-chain, dynamic interventions, especially the use of unstable surfaces, have been proved in many orthopaedic disorders such as patients with chronic ankle instability. ${ }^{17}$ In this context, it is worthwhile to consider not only the extrinsic foot muscles, but also the intrinsic foot muscles. In fact, there is a growing body of evidence that the role of these muscles has been underappreciated, especially in the perspective of regaining normal extremity movement patterns. The paradigm which is addressing this relationship has been called "foot core system". ${ }^{18}$

Our study has several limitations. First, the study population is small which affects the statistical power of the study. Second, we chose to examine balance control only during a transition task from double-leg stance to single-leg stance. It is assumed that this task is more challenging for the postural control system when compared to other balance tasks (eg quiet standing with EO and EC or single-leg standing) and that therefore, it faces higher sensitivity in detecting postural impairment in patients. However, a more comprehensive perspective would have been provided if also other balance tasks or alternative testing procedures were included, especially as other studies found considerable within subject differences. Interpretation of the current findings should therefore be performed with some caution and in light of these flaws.

\section{5 | CONCLUSION}

The current study aimed at unravelling the balance ability of children and adolescents with haemophiliac with and without 


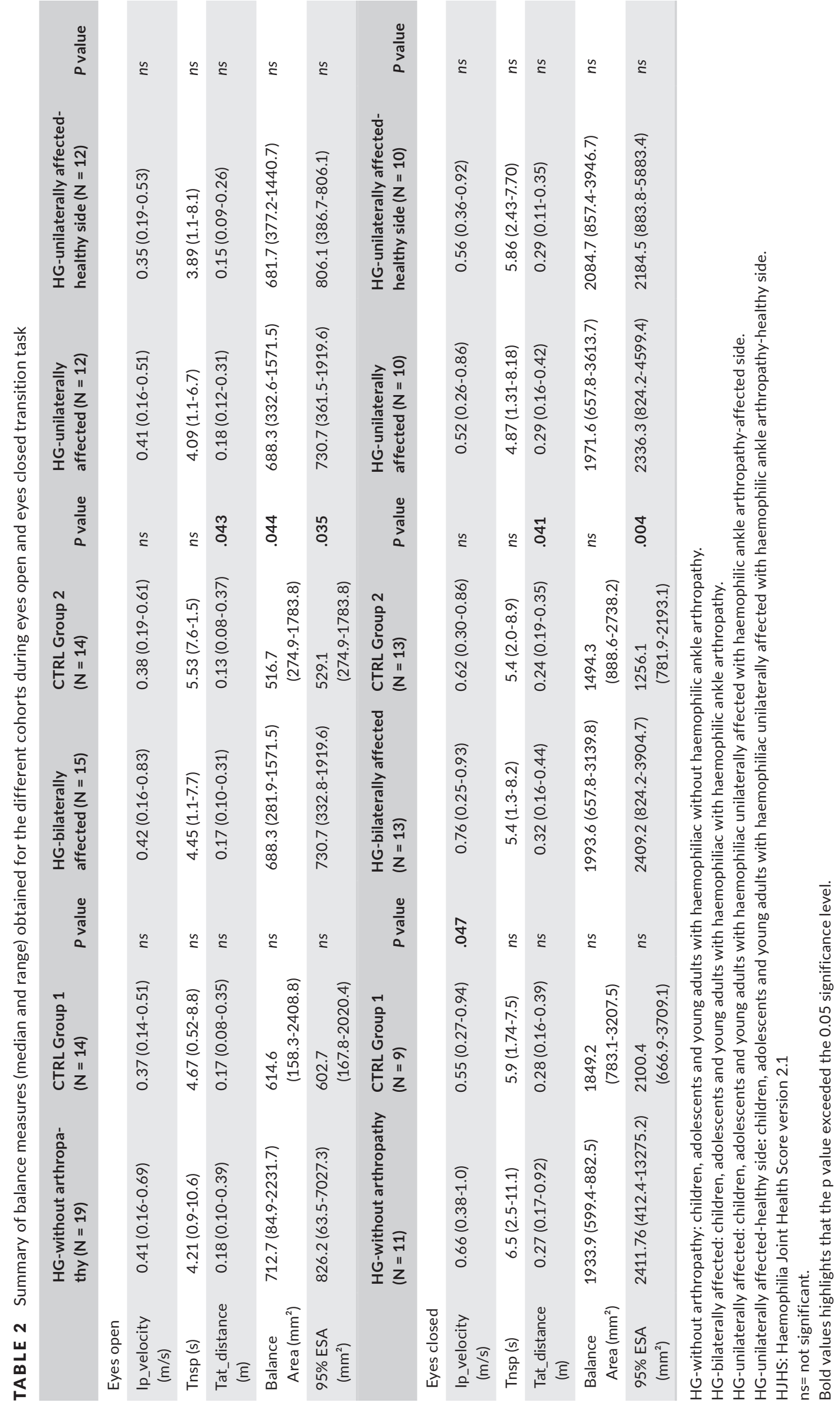


MRI-based signs of ankle arthropathy. Based on the findings of the current study, it is suggested that chronic bleeding episodes initiate a complex pathophysiological including neurophysiological modulation which may originate both from unilateral and from bilateral deterioration of the musculoskeletal system. As recommended by the World Health Organization, joint assessment should be performed according to the International Classification of Functioning, Disability and Health (ICF, 2001). The "body function and structure" area of this field is traditionally evaluated using radiological and clinical joint score. While radiological scores only provide information about the structure, clinical joint assessment integrates both components. Functional performance is therefore multifactorial. The results of the current study highlights, once more, that implementing biomechanical assessment tools and developing research protocols based on the evaluation of muscle and joint function will provide a stronger basis for the management of patients with haemophiliac.

\section{ACKNOWLEDGEMENTS}

Authors are grateful to Dr. Bart Dingenen for helpful comments during the data analysis as well as to Ir. Luc Janssens and Dirk Desmet for their technical support. The authors are also grateful to Prof. Dr. Jean-Baptiste Pialat and Dr Thomas Brunel (Department of radiology, Hôpital Edouard Herriot, Hospices Civils de Lyon, Lyon, France) for scoring the MRI examinations of all CA\&YAwH. This study was supported by the ASPIRE 2013 (Pfizer) research grant (KD), by the World Federation of Haemophilia Clinical Research Grant Program (KD, SL) as well as by the Bayer Hemophilia Awards Program (Early Career Investigator Award 2012) (SL).

\section{DISCLOSURES}

The authors stated that they had no interests which might be perceived as posing a conflict or bias.

\section{ORCID}

K. Deschamps (iD http://orcid.org/0000-0001-6559-6237

S. Lobet (iD http://orcid.org/0000-0002-3829-6850

\section{REFERENCES}

1. Lwaleed BA, Bass PS. Tissue factor pathway inhibitor: structure, biology and involvement in disease. J Pathol. 2006;208:327-339.

2. Roosendaal G, Lafeber FPJG. Blood-induced joint damage in hemophilia. Semin Thromb Hemost. 2003;29:37-42.
3. Coppola A, Tagliaferri A, Di Capua M, Franchini M. Prophylaxis in children with hemophilia: evidence-based achievements, old and new challenges. Semin Thromb Hemost. 2012;38:79-94.

4. Oldenburg J. Optimal treatment strategies for hemophilia : achievements and limitations of current prophylactic regimens. Blood. 2015;125:2038-2045

5. Gauchard GC, Vançon G, Meyer P, Mainard D, Perrin PP. On the role of knee joint in balance control and postural strategies: Effects of total knee replacement in elderly subjects with knee osteoarthritis. Gait Posture 2010;32:155-160.

6. Hatton AL, Kemp JL, Brauer SG, Clark RA, Crossley KM. Impairment of dynamic single-leg balance performance in individuals with hip chondropathy. Arthritis Care Res. 2014;66:709-716.

7. Fearn M, Hill K, Williams S, et al. Balance dysfunction in adults with haemophilia. Haemophilia. 2010;16:606-614.

8. Gallach JE, Querol F, González LM, Pardo A, Aznar JAPosturographic analysis of balance control in patients with haemophilic arthropathy. Haemophilia. 2008;14:329-335.

9. Souza FMB, McLaughlin P, Pereira RP, et al. The effects of repetitive haemarthrosis on postural balance in children with haemophilia. Haemophilia. 2013;19:e212-e217.

10. Boccalandro E, Pasta G, Mannucci PM, et al. Integrated postural analysis in children with haemophilia. Haemophilia. 2014;20:263-267.

11. Hilberg T, Herbsleb M, Gabriel HHW, Jeschke D, Schramm W. Proprioception and isometric muscular strength in haemophilic subjects. Haemophilia. 2001;7:582-588.

12. Brunel T, Lobet S, Deschamps $\mathrm{K}$, et al. Reliability and clinical features associated with the IPSG MRI tibiotalar and subtalar joint scores in children, adolescents and young adults with haemophilia. Haemophilia. 2017;24:141-148.

13. Lundin B, Manco-Johnson ML, Ignas DM, et al. An MRI scale for assessment of haemophilic arthropathy from the International Prophylaxis Study Group. Haemophilia. 2012;18:962-970.

14. Foppen W, van der Schaaf IC, Witkamp TD, Fischer K. Is joint effusion on MRI specific for haemophilia. Haemophilia. 2014;20:582-586.

15. Dingenen B, Staes FF, Janssens L. A new method to analyze postural stability during a transition task from double-leg stance to single-leg stance. J Biomech. 2013;46:2213-2219.

16. Dingenen B, Janssens L, Luyckx T, Claes S, Bellemans J, Staes FF. Postural stability during the transition from double-leg stance to single-leg stance in anterior cruciate ligament injured subjects. Clin Biomech. 2015;30:283-289.

17. Webster KA, Gribble P. Functional rehabilitation interventions for chronic ankle instability: a systematic review. J Sport Rehabil. 2010;19:98-114.

18. McKeon PO, Hertel J, Bramble D, Davis I. The foot core system: a new paradigm for understanding intrinsic foot muscle function. $\mathrm{Br} J$ Sports Med. 2015;49:290-299.

How to cite this article: Deschamps K, Staes F, Eerdekens M, et al. Postural control during a transition task in haemophilic children, adolescents and young adults with haemophilic ankle arthropathy. Haemophilia. 2018;00:1-8.

https://doi.org/10.1111/hae.13484 